\title{
Dyslexia in the University. Guidelines for inclusion and teaching of the University of Florence
}

Tamara Zappaterra ${ }^{1}$

\begin{abstract}
This paper reports the results of an interdisciplinary project (pedagogical, medical, engineering area) of the Centre for Research on Disability Issues, operating within the University of Florence, which provides support for students with disabilities and with Learning Disorders (LD). The project, which aims at drawing up a protocol for the inclusion and learning support for students with learning difficulties, after a first reconnaissance of user needs that have been reached with the participation of the very same protagonists (thanks to a questionnaire built on purpose and a series of focus groups) is now at the stage of guidelines drafting for inclusion and education, to be used by teachers, students and administrative staff. In the article, after a brief summary of the previous phases of the project, the reader can find the most significant elements of the guidelines for teaching, i.e. the section for teachers, whose aim is to implement a LD-friendly teaching, even at the university level, in compliance with Law 170/2010.
\end{abstract}

Keywords: Learning Disabilities, Inclusive Education, Special Education Needs, Didactic tools, University.

\section{Dyslexia as a developmental disorder}

Dyslexia and other LD (dysgraphia, dyscalculia and dysorthography) are organic-based neurological disorders with an evolutionary trend. This means that the affected capacities of reading and writing appropriately, in addition to capacity of numerical cognition, appear both as a slowing down and/or an atypia with respect to the norm, and present characteristics that vary depending on the age of the subject and can also vary according to the type and nature of support that can be provided to the subject (Dehaene, 2009; Gérard, 2011).

The international research has focused its analysis on the characteristics of LD in childhood, which is when they show for the first time their evidence in the impact with the primary school. Therefore, we know quite well the difficulties that a 6-10 years student meets compared to the demands at school, but we are still far from a thorough understanding of the nature and

${ }^{1}$ University of Florece. 
characteristics of such disorders in adolescence and adulthood (Hatcher et al., 2002; Reid et al., 2006, Genovese E. et al., 2011).

There are various kind of reasons: sometimes these students have not been identified as dyslexic in childhood; this means they have escaped to an early diagnosis, finding compensation to their difficulties autonomously, so the school does not recognize them as dyslexic students and they are not even aware of it. Sometimes, even the secondary school teachers are not able to recognize the specific disorders of the students and confuse it, as it happened until the recent past, with negligence or little desire to study. This is indeed a problem due to the lack of knowledge of such disorders in school, especially primary and secondary, and is in short a precise problem of teacher training.

Italy has come with some years of delay -compared to the international scenario- to address the issue of LD in schools, probably because of the intrinsic nature of the Italian language that has a transparent spelling basis. This means that the almost perfect correspondence between grapheme and phoneme in the Italian language raises the bar -or the special needs of an Italian dyslexic student- at a higher level compared to what happens in countries with an opaque orthography. This does not mean that Italy has less dyslexics than other countries; rather, in our national context, the difficulty threshold, compared to the demands of school environment, can show its evidence later, in less severe situations, when the nature of the educational demand and cognitive burden for students become greater.

Such issues become even more urgent in the university context. Spelling, meaning, and appropriate use of the specific vocabulary of the various disciplines represent an additional challenge for the dyslexic student. Moreover, even at this age group, the psychological consequences of the difficulties related to the LD are important. The dyslexic adult captures, more and better than the dyslexic child, the differences between his performances and those of others, thus studying harder, putting more effort and commitment to succeed in profit. Sometimes, when the effort seems enormous and there is an incomprehension by the teachers and the family, the school dropout or the choice of not continuing the studies up to university level is the result of a renounce due to excessive increase in workload and in the amount of materials to read (Lami et al., 2008; Zappaterra, 2012; Trisciuzzi and Zappaterra, 2012, 2014).

However, even late diagnosis and appropriate interventions in secondary school and in University allow these students to achieve brilliant results. Yet, teachers shall take into account the developmental characteristics of the disorder: if in primary school it is important for the pupil to read fluently and correctly and to understand the text, in secondary school and university the method of study becomes the priority, as well as the implementation of a teaching in LD-friendly mode, since the work load is considerable. It is thus 
necessary to find, with the student, the most effective strategies to study the different subjects, introducing, where necessary, compensatory instruments such as digital books, speech synthesizers and conceptual maps software that enable the student to be autonomous in the study.

If the school is asked to give a strong contribution in this direction by drawing up a personalized educational plan, covering the most suitable adaptations for each pupil with LD, all this, inside the Universities, must be requested autonomously by the student. The university, as well, is preparing a shared reflection for the inclusion and the adaptation of teaching to the specific needs of dyslexic university students.

\section{The research project on LD and University2}

This is the context in which can be introduced the research project "Students with LD at University. Implementation of a methodological protocol for the usability of teaching and self-study" currently underway at the University of Florence, promoted by the Centre for Research on Disability Issues (CESPD) and sponsored by the Ente Cassa di Risparmio di Firenze. The project, started in 2015, places itself within the framework of the actions aiming at ensuring the right to study in universities for students with LD, and at creating an educational-management protocol for inclusion of students with LD in the University of Florence.

Since 2010 -the issue year of the Italian law that protect LD- in fact, there has been a yearly exponential increase of LD students in the University of Florence. This is undoubtedly the sign of a gradual dissemination of knowledge of such disorder, and greater awareness of the rights of these students. However, this should also result in welcoming activities, but, above all, in quality interventions on teaching and evaluation, that, while respecting the special educational needs of LD students, at the same time are able to safeguard the disciplinary contents and training objectives that characterize the courses of study and the corresponding final professional profiles.

Such goal has to be kept always in consideration when addressing the university context, because while the school has the duty to organize the teaching for the entire class group, taking into account the specificities of LD pupils (who could be, according to data from Ministry of Education and University, an average of two for each class), the University must safeguard the

2 This paragraph is most inspired by Zecchi-Orlandini S., Zappaterra T., Campatelli G., Ariani L., Meneghin A., Rossi C., Zudetich C., "Students with Learning Disabilities at University. Design of a Protocol for Usability of Teaching and Individual Study", Italian Journal of Special Education for Inclusion, 1, 2015, 125-136. 
epistemological and fundamental aspects of the disciplines that characterize the specific training and professional profile of the different degree programs, and it is the student who shall adapt to this, particularly by adopting a personal method of study. This does not prevent university teachers to use certain tricks, even educational; but these elements are placed inside a program of personalization of the course of study, that takes place in an exclusive relationship between teacher and student.

The first phase of the project was devoted to the study of the present situation regarding the management of university students at the University as it arises from the literature, with a particular focus on Italian University, considering the peculiarity of our language. The second phase involved the investigation about the user needs: thanks to a specific questionnaire prepared on purpose and then through a series of focus groups, the nature and characteristics of the needs of university LD students in the university course have been investigated, directly by interviewing the students of the University of Florence. At the beginning, the project (which is currently underway) involved 43 students, who now are, after two years, over 50.

Later, the research team considered appropriate to organize the following phases of work orienting them in two directions:

- Development of university guidelines for taking care of the university career of the LD student by all the actors involved (academic staff, technical and administrative staff, services, students, etc.), expecting that such guidelines can offer good practices and operational guidance to the University, and be useful in order to support the university study program of the students, the educational offer, the teaching activity of teachers, promoting greater effectiveness and efficiency of university facilities intended for students with LD;

- Creation of a web-space within the university website on the basis of usability criteria declined according to the specific needs of LD students, and addressing students, teachers and administrative staff of the University of Florence, expecting such website to be in practical terms a means able to provide information and/or directions in relation to facilities, applications and supporting materials made available by the university system, in order to accompany the entire university career of the student with $\mathrm{LD}$, from the entrance exam to the achievement of the degree. This web space will be available and directly accessible online by the user.

Eight thematic sections compose the survey about the previous university experience of students with LD:

1. "Identity records (personal data)", in which are asked for information about age, sex, year of graduation, degree, diploma and any other kind of previous educational experience; 
2. "Diagnosis and Intervention", in which are asked type and age of diagnosis, including reporting of any co-morbidity. In this section, students are also asked to report any other contact with associations of LD protection;

3. "Relations with the university", in which students are asked to evaluate in terms of quality, and always in relation to their LD, their experiences occurred during university lectures, examinations and entrance exam, and while using didactical materials, university website and forms, both on paper and online;

4. "Relational dimension", in which the students are asked to express their opinion on the quality of the relationships established in the university environment, with teachers, administrative and technical staff and fellow students, in relation to their particular needs of LD student;

5. "Support provided by university", where the students are asked to assess the level of knowledge and satisfaction experienced in the use of the main support facilities intended for LD students in the Florence campus, with particular reference to Centre for Research on Disability Issues (CESPD), mentoring and others facilitator instruments;

6. "Instruments", in which are asked information about technological tools and computer software aimed at supporting LD students, used during their school and university career, and the degree of satisfaction achieved in their use;

7. "Individual Strategies", where students are asked to highlight and evaluate the effectiveness of strategies used to improve learning capacity, with respect to the specific difficulty arising from their condition of LD students;

8. "Feedback", where students were asked to express an opinion on the questionnaire itself with regard to the specific issues of LD student, giving an evaluation of both the relevance of the content and the adequacy of the choices made about language, graphics and type of digital interface used.

The eight sections described above have been divided into twenty-four questions including, where possible, the use of multiple-choice quiz, and only when strictly necessary, open-ended questions. The precise combination of these two different ways of formulating questions allowed to achieve standard answers that can be easily grouped and quantified, but also to collect examples, motivations and personal observations of students otherwise undetectable.

Where the goal was the recognition of a degree of satisfaction, we have constantly provided the use of closed questions (multiple choice) on four levels of evaluation (not at all; little; enough; very much) formulating the question so that the choice of a response with increasing amounts always corresponded to a shift of opinion toward a positive value.

For the creation of the online survey we used the "Forms" function in the free version of Google Drive trying to use the various options offered by the program for the construction of an interface as much as possible LD-friendly. Sixty 
percent of students responded to the questionnaire. The result is an average age of students of approximately 21 years, with the first diagnosis of LD received at around 12 years. Only half declares to be in contact with associations of LD protection. Moreover, despite the small sample size, the percentage of responses received from students enrolled in the School of Liberal Arts and Education (46\%) turns out to be significant, outdistancing the students belonging to other faculties, clearly highlighting how this field of study -strictly connected to their personal problems- represents the first choice for LD students.

The following aspects of university life were immediately detected as particularly critical:

- the use of the website of the University of Florence;

- the use of the online form;

- the use of the paper forms;

- the use of test tracks and examinations.

Students, in general, perceive as positive the personal relationships developed during university experience. The highest levels of deficiency in facilities provided by the university resulted in the use of customized study locations and in the service of textbooks digitalization.

As far as the use of technological support tools is concerned, a poor using experience of them has emerged, with the only exceptions regarding personal computers and smartphone. Also significant are the data related to individual strategies developed by students during their studies, which showed overall a lack of knowledge of support strategies. A substantial appreciation of the questionnaire results by the analysis of feedback data on such tool.

After administering and analyzing the questionnaire results, the investigations on the students with specific learning needs continued with the implementation of four focus groups. The purpose was to investigate some of the "critical issues", which were perceived as a priority by the students and at the same time resulted as potential elements to intervene on concretely, in order to keep working on LD Project.

Accordingly, the following areas of analysis have been identified:

1. The University website that, in the question about the satisfaction degree of student with LD, regarding the various aspects related to their university life, reached the lowest average value overall, that is 1.88 . The decision to carry out a further study on the website is also due to the fact that one of the outcome of the project will be the LD-friendly web site;

2. The relationship with professors, that although judged satisfactory both in terms of interpersonal relationships (average value of 2.88), and in the support tools for teaching (slides projection in classes: 2.64, digital material provided: 2.70 and draft for examinations: 14.2), had the highest number of reports in the open answers, thus revealing a widespread heterogeneity in behavior; 
3. The support tools for teaching, in which the most obvious fact was not so much the level of satisfaction of use, but rather the low level of knowledge and use by students themselves, in many cases lower than $50 \%$ of the total of students responding to the questionnaire;

4. The support facilities that the University provides for students with LD, often not known also in this case and, when known, sometimes judged as unsatisfying.

For the data collection during focus groups it was decided to resort to a typical classical method of Quality Function Deployment (QFD), a tool originally used for the management of corporate quality, whose versatility makes it easy to use, however, also in extremely different contexts. In fact, since each set of objects and people interacting with each other can be considered as a system and that any sequence of activities oriented toward a goal can be considered a process, it follows that the theoretical bases and methods developed by engineering quality can become functional also in original and heterogeneous environments.

In particular, the method used was that of the simplified correlation matrix, a grid in which after identifying the customer, in this case the students participating to focus group, themselves, under the guide of the moderators, identify the critical elements (the so-called VOC, voice of costumer) related to the scope of analysis, and define possible solutions (the so-called CTQ, critical to quality). The result is a matrix in which each VOC is associated to one or more CTQ. During the four focus groups, moderators and students have therefore worked in teams in order to build four correlation matrices, one for each analyzed areas. The construction of the matrix has been made in real time, projecting it so that it was visible to all.

After choosing the topics and the method of data collection, it was decided to limit the working time of each focus group to 30 minutes, including introduction, collection and reorganization of VOC (in order to eliminate repetitions, irrelevant items, etc.), collection of CTQ and final construction of the matrices. Obviously, during a following debriefing carried out by the moderators group only, all the matrices were further arranged with a better organization.

Two moderators, two observers of the Work Team and eight students attended each focus group. Such number is considered suitable, based on statistical studies available at present, in order to determine at least $90 \%$ of the relationship problems among users of a system and the system itself.

The analysis of the correlation matrix allowed directing the following activities of the project towards specific lines of intervention, derived from the analysis of CTQ. In particular, we may summarize the main results as reported below: 
1. The laws alone are not sufficient to ensure LD students full integration within their university career. The rules must be associated to guidelines and handbook of good practice;

2. It is necessary to invest in training processes and information of all those involved; such process shall be used in order to "educate" all users to relate properly with each other and with the educational and compensatory instruments available;

3. The statement of the previous paragraph shall facilitate the realization of a parameter of behavioral "homogeneity" in the relationship between teachers and students. In short: teachers and students need to know what they can get from one another, but also what the limits of their requests are;

4. A website collecting guidelines and handbook of good behavioral practice is necessary but it must be structured on several interpretation levels (for teachers, for students, and for university staff that relates to the phenomenon of LD) and, above all, it must be user-friendly for the "weakest" users, with ad hoc contents and layout;

5. It is fundamental to provide user-friendly compensatory instruments, not bound to that technological obsolescence that would soon cause them to be useless.

\section{Guidelines for inclusion and education for teachers use}

Once concluded the phase of user needs investigation, the research team crosschecked data taken from students with methodological didactical and regulatory guidance, available in the specific bibliography about the subject. Guidelines for the inclusion and education for students with LD have thus been drawn up, to be used by teachers, students and technical and administrative staff. This paragraph shows the sections that make up the guidelines, in the specific section intended for professors. This part is made of six sections, as follows:

1. The nature and specificity of the $L D$.

The first section gives teachers a basic understanding of the specificity of the LD through an Information Box called "LD keynotes", which highlights the specific nature of disorders, which preserve the intellectual functioning and are developmental, as they vary in function of the age of the subject and have a significant impact on the ability to adapt to the study.

We find then a Legislative Box named "Reference standards for the management of students with LD in the university", which reports the recommendations provided by Law 170/2010, by the implementing decree 5669 of the year 2011 and by the Ministry of Education and University Guidelines. It is underlined the way in which the guidelines attached to the Ministerial Decree No. 5669 of 2011 explain how to safeguard the right to study. 
Such elements are further explained in the Box about "Good practice in managing the use of compensatory instruments and dispensatory measures" also considering the CNUDD (University National Conference of Delegates on Disability) guidelines that have been recently integrated with a section regarding the LD (CNUDD, Conferenza Nazionale Universitaria Delegati per la Disabilità, Linee guida del 10 luglio 2014, http://tinyurl.com/nvcw2yk). It is important to remember that the law does not give information on the submission time of application for compensatory instruments and dispensatory measures by the student. A good practice could be, for the teacher, to make a brief notice about it during the course introduction, also placing such communication for LD students on its web space, inviting them to agree in private and within a deadline the requests for dispensatory forms or compensatory measures of which they can benefit during exams and classes.

\section{Production of didactical and computer materials for $L D$ students}

This section explains how to facilitate the text readability, the graphic layout and the page setting parameters as well as font, style, spacing, size, color and background, the page organization, and the use and construction of conceptual maps. Experts say that there is no "universal" font, which can adapt to the specific needs of all readers with LD. However, over the time several types of DSA-friendly font have been proposed and tested. For example, Dyslexie and OpenDyslexic fonts use heavy lines, alternating different heights and semi-italic inclinations, in order to try simplifying the identification of the each letter (Reid, Strnadová and Cumming, 2013; BDA, 2015).

Following, the section provides an information box on Universal Design for Learning. The Universal Design (UD) is an innovative approach to the design of locations, buildings and tools, aiming at making them available and accessible to all categories of persons, with a wide extension and without the need for special adjustments or solutions (CAST, 2011; Meyer et al., 2014). Briefly, the basic idea is that of a design performed according to the UD standards, so that everyone can benefit from it, regardless of personal characteristics (age, ability, personal situation, and then, obviously, the presence of disability).

The principles on which the UD is based are:

1. Fairness - fair use: anyone can use it;

2. Flexibility - flexible use: it adjusts to different abilities;

3. Simplicity - easy and intuitive use: easy to understand;

4. Perceptibility - the ability to actually transmit sensorial information;

5. Tolerance to errors - minimizing risks or unintended actions;

6. Containment of physical effort - use with the smallest effort;

7. Satisfying sizes and spaces - making the space suitable for access and use.

The Universal Design for Learning (UDL) adopts the same design standards in the area of education. The aim is to change the methods of contents administration, so that they do not only pertain to the "average student" (to whom curricula and educational tools are usually addressed) but also include marginal students, that is those with learning difficulties, but also -and it may seem a paradox- those particularly talented.

The specific principles on which the UDL is based are:

1. Providing multiple means of representation;

2. Providing several means of action and expression;

3. Providing several ways to support commitment.

For students with LD, guidelines are declined in the need to use tools that facilitate their involvement, but at the same time prove to be useful also for all other students. Typical 
examples are the use of conceptual maps and alternative media tools (videos, eBooks, slides etc.).

As far as accessibility and usability of web texts are concerned, the Italian relevant legislation is Law no. 4 of January $9^{\text {th }}, 2004$, the so-called "Stanca Law", which makes it mandatory to design public sites (or public interest sites) in accordance with the principles of accessibility. The law implements the principle of equality established by art. 3 of the Italian Constitution, guaranteeing the right of access to public administration computer and data services also for people with disabilities.

With Ministerial Decree of March 20 th 2013 the attachment n. 2 on "Technical requirements and different levels of accessibility to ICT tools" has been amended and updated in order to harmonize the Italian law with the provisions of WCAG 1.0 and WCAG 2.0. (Web Content Accessibility Guidelines). The latter are the guidelines for accessibility to Web contents, defined by the W3C (World Wide Web Consortium) offering a standardized accessibility criteria.

The law ISO 9241, published in 1998, defines usability at an international level. In particular, ISO-9126 refers to usability such as facility of use, learning, understanding and enjoyment of a software.

With respect to the adaptation of texts to reading software, it is specified how texts (lecture notes, presentations, etc.) can be adjusted to reading software, making them available in electronic format, but also drafting them in a format that allows a correct interpretation by the software. This can facilitate learning both for students with LD and those with impaired vision.

It can be stated that:

-.doc files (and similar) are the most suitable, because they are adjustable to the individual viewing preferences, and better adaptable to the needs of the reading software;

-.pdf files maintain more effectively their original formatting style, but their "stiffness" sometimes makes them not fully usable by reading software.

3. Organizing lessons for $L D$ students and structuring a $L D$-friendly lesson

Few simple solutions by the teacher could considerably facilitate the learning process of a student with LD (Weingarten Learning Resources Center, University of Pennsylvania, 2002; Disabled Students' Program, Berkeley, University of California, 2011; aDShe, 2011; The Ohio State University Partnership Grant, 2015; DO-IT, University of Washington, 2015). In general, in order to adapt the lesson to a class that includes people with LD, the teacher should include:

- the use of different codes to convey information and knowledge, as any deficiency could relate to different learning processes;

- the activation of frequent feedback for checking what has actually been transmitted;

- the provision of notes, index and various types of didactical materials that can facilitate the acquisition and systematization of information;

- the exemption from the need to take comprehensive notes during class.

Moreover, some extra time should always be provided in case of need for clarification and systematization of the above-mentioned elements. A good practice would be to divide each lesson in several sections of limited duration, corresponding to a single theme/topic, separated by short breaks; at the end of each section, the professor should provide a summary of the subject, as well as some time for questions.

In order to facilitate the learning process of a student with LD, it is recommended for the teacher, during the first part of the lesson, to provide an index and/or an orderly track of the topics that will be discussed with particular reference to the general study program. 
Such practice can facilitate note taking, understandable also after a long time, and can encourage the structuring of the learning process, making it easier for the student to focus on the content of the lesson.

When possible, the index and/or draft should be available for students before classes. Such notes should include bibliographic references, in order to allow any further reading for those who desire to deepen the knowledge of the topic. A good practice would be that of placing on the web space of the course, before the beginning of the lessons, all the index, tracks and bibliography of each lesson, still presenting them in the classroom before any lesson. If necessary, the teacher could also consider the possibility to present the lesson content in form of conceptual map, using it during the lesson as index and summary tool.

The use of different forms of communication and representation during classes could be very helpful for LD students (as well as for everyone else), who could use their full potential in order to acquire information through the process that is most suitable for them, thus compensating their difficulties. The teacher should present the most important information and the essential concepts in both written and oral form. It would also be useful, during the classes, to read aloud the blackboard and the slides contents. These precautions should be taken also when providing the various practical information about the course (bibliography, events, programs, etc.).

If possible, it is recommended to include in the lesson all forms of synthetic representation (illustrations, plans, charts, pictures, tables and conceptual maps), in order to explain graphically all the concepts and information.

As for the lesson fruition, students with LD should be exempted from taking comprehensive notes during classes, so that they can properly concentrate on the content of the lesson. Consequently, they should have the possibility to:

- $\quad$-record the lesson;

- -take pictures or reproduce in any other quick way what has been done in the classroom on the blackboard;

- -take pictures or reproduce in any other quick way what has been presented in the classroom by the teacher through the slides.

Some of the material (slides, exercises, notes and handouts of the course) could be made available by the teacher, possibly in digital format, so that it can be used by students also through the appropriate compensatory measures.

From a legal point of view, teachers and students must take into account that recording lectures is allowed for individual study purposes and is not included in the legislation on privacy. Guidelines for the right to education of students with LD, referred to in the implementing Decree n. 5669, provide for the ability to record the lessons in order to facilitate learning. The text reads: "The Law 170/2010 also calls the institutions that deal with education and learning to the duty of ensuring the introduction of compensatory measures, including alternative means of learning and information technologies, as well as dispensatory measures for some performance which are not essential for the quality of the concepts to learn".

Not all teachers are aware of these rules and know what they can do to facilitate selfstudy without their privacy being injured. Given the current legislation, it should also be noted that the recording of lessons for individual study purposes does not pertain to legislation on privacy. The Authority on Privacy, consulted on the issue, in fact, reports that: "[...] In this regard, this Authority has specified that students can record lectures when the registration is done for personal purposes, such as for reasons of self-study. For any other different use or any distribution, even on internet, on the contrary, it is necessary to inform properly the people involved in the recording (professors, students 
...), and obtain their explicit consent". On this and other matters, useful information can be found in the brochures [...] published by the Authority for the protection of personal data, which can be downloaded in electronic format from the Authority's website www.gpdp.it at the following link http://tinyurl.com/ow9n5fd.

With regard to slides construction, the main criteria to which a teacher can refer for the creation and use of effective slides is that of preferring an exposure of the concepts through bulleted/numbered lists and keywords. The use of short texts allows the teacher to explain easily the contents during class, referring to the content of the slides as an index or a summary. From a graphical point of view, in order to facilitate the readability of the slides content, their design should be as logical as possible, simple and clear, and both text and images should be sufficiently big and spaced. In particular, when creating the slides, it is recommended to pay particular attention to the following aspects (Rello and Yates, 2012; Rello et al., 2013; Aiutodislessia, 2013; BDA 2015):

- Combination of colours: avoid too little contrasting effects between the texts, images and the background (e.g. Yellow text on a white background); avoid too much contrasting effects between texts, images and the background (e.g. black text on a bright white background); use blacks (or dark) character on a light grey or pastel background.

- Text Character: avoid unusual or special characters, and choose the type "sans serif" (suggested: Arial, Comic Sans, Helvetica, Tahoma and Verdana);

- Size and line spacing of the text: use of minimum font size of 18 points (preferably 24 or 28); use at least 1.5 line spacing;

- Text emphasizing: give priority to the use of bold; use the underlined only for hyperlinks; avoid the use of italics and capital letters; use with discretion colour as a form of text highlighting.

\section{Exams management for students with $L D$}

Students with LD are entitled to a personalized treatment during examination, possibly also through specific technological aids. The adaptation of the tests shall be agreed in advance with teachers with reference to the provisions of the "Guidelines for the right to education of pupils and students with LD, attached to Ministerial Decree of July $12^{\text {th }}$, 2011". What can be adapted is exclusively the form of the test: the degree of difficulty of the test and the preparation required to the candidate must be similar to that of all other students.

Given the great variability of the manifestations of LD and considering the different characteristics of students, the alternative forms of examination and/or the use of any compensatory tools or dispensatory measures should be assessed individually according to the type of diagnosis and in relation to specificities of the individual disciplines and the purposes of study courses.

The most appropriate form for such an assessment is a private conversation between teacher and student, held well in advance of the exam, for an in depth examination of personal diagnosis and specific difficulties of the student. In case of need, both the teacher and the student may request the support of the Disability and LD representative or of the CESPD (Centre for Research on Disability Issues), which can eventually send a tutor.

A good practice for the teacher, which could considerably facilitate the management of examinations of LD students, is to inform at the beginning of the course about examination timing, contents and operating methods, explaining what could be the subject of examination, the evaluation criteria and the test methods. On that occasion, 
the teacher could also address a general call to students with LD -who believe to be in need of an adjustment- to ask for a private conversation before a given deadline.

Another good practice is that of making available, well in advance of the exam, tests, bibliography and any handouts, since the LD student may need extra time to convert paper texts in digital format.

Written vs. oral. All types of LD tend to reduce the capacity to carry out correctly and quickly a written test. Tricks and various kind of help enable to compensate adequately such difficulties. However, if the type of discipline and organization of the course make it possible, it could be advisable to organize an oral examination for the student with LD.

It is recommended, for the candidate, to access oral examination without being preliminarily subjected to an "attempt" to written test, whose likely consequence would only be the creation of a sense of frustration and a useless waste of time. In the event of an indispensable written examination, the teacher should carefully evaluate the most appropriate form of the tests, in order to make them as accessible as possible to a LD student.

Some precautionary measures can help a student with LD to perform the written test:

- -performing the examination in a quiet area, as it could be difficult for a student with LD to interpret a text when there is a background noise;

- - - using lined or arithmetic exercise sheets, instead of white blank page, in order to be able to organize better and more easily text and figures;

- $\quad$-using rough copies, in order to reduce the stress producing immediately a tidy and definitive text.

Extra time. For a LD student fast reading, fast writing, and the rapid and systematic information retrieval can be difficult. However, in many cases the process of decoding, writing or information retrieval may be carried out correctly if performed with additional time. In such cases, it would be appropriate to provide, for students who will have extra time, a sufficiently secluded area. Even during an oral examination, it could be effective allowing additional time to a LD student, in order to answer all the questions.

The possibility to gain access to examples and draft of old exams in reasonable advance, can help the student to carry out properly the exam or at least to identify and agree on time with the teacher the granting of appropriate compensation tools and dispensatory measures.

The recommendation of the Section of Guidelines by the CNUDD (University National Conference of Delegates on Disability) of July 10th, 2014 dedicated to LD report the following dispensatory measures and compensatory instruments:

\section{Dispensatory measures}

- Considering the possibility to split the examination subject in several partial tests;

- Privileging oral rather than written exams, taking into account the individual skills profile;

- Whereas the written examination is considered necessary, check whether the chosen format (e.g. multiple choice test), may represent an obstacle, and whether it can be replaced by other forms of written evaluation;

- During the written tests, provide a quantitative -but not qualitative- reduction of the test, or extra time up to a maximum of $30 \%$, for carrying out the test;

- In the evaluation, consider contents rather than form and spelling.

Compensatory instruments

- Digital recorder;

- Texts in digital format; 
- Speech synthesizer programs;

- The presence of tutor with a reader function, in case it is not possible to provide materials in digital format;

- Calculator;

- Tables and forms;

- Conceptual maps;

- Didactical materials in accessible formats (presentations, handouts, exercises), provided, if necessary, before lessons;

- Other technological facilitation instruments in the phase of study and examination.

\section{Admission test management for students with $L D$}

The implementing guidelines of the law 170/2010 article 6 paragraph 7 state that for a person with LD in the university "The submission, at registration, of the diagnostic certification, enables to access the admission test with the following procedures:

- The granting of additional time, with respect to those established for all other students, judged suitable by the University in relation to the type of test and, in any case, not more than $30 \%$ extra time;

- The granting of additional time up to a maximum of $30 \%$ more than that established for the admission tests to undergraduate and master's degree planned at national level or by University in accordance with art. 4 of the Law of August $2^{\text {nd }}, 1999$ n. 264;

In cases of a particularly serious certification of LD, the universities -in their autonomymay consider further measures intended to guarantee equal opportunities in the tests performance."

Ministerial Decree 463 of July 7th, 2015 regarding admission tests reaffirmed (Article 11 paragraph 2) what stated by law about the requirement, for applicants with LD, of proper certification submission in order to be able to make use of compensatory instruments, setting the additional time granted to them as $30 \%$ more (the maximum amount allowed by law 170/2010).

In order to ensure the right to education of a candidate with LD, the selection board of an admission test and knowledge verification must:

- Check, with the assistants help, the presence of candidates with certified LD and their possible requirements;

- Check which compensatory measures must be provided according to the announcement and the certification of each candidate with LD;

- If necessary, refer to the August 6th, 2015 communication of CNUDD (University National Conference of Delegates on Disability) that defines as eligible those compensatory instruments "that allow the mere performing of solving processes previously identified independently by the candidate" excluding "those who would ultimately constitute an unjust facilitation -in respect of the other candidates- during the identification of the correct answer";

- Make sure it is logistically possible to grant the use of expected compensatory instruments during the tests;

- Contact the Department or School Delegate for Disability for any explanations, feedback or help in the management of organizational aspects;

- Contact in advance the CESPD (Centre for Research on Disability Issues) for possible explanations, evaluations or help in the management of organizational aspects;

- Always keep in mind that the use of compensatory instruments during tests is a right of the candidate with LD, granted by the law. 


\section{Further resources for $L D$ students}

The Guidelines end with the section containing information about the University facilities that can be contacted (CESPD-Centre for Research on Disability Issues), the names of the Deputy Rector on Disability and LD and the same Deputies in Schools and Departments. In addition to the delegates, the University of Florence has provided students with LD with a web site. On the University website, in fact, there is a section entirely dedicated to the management of LD students at the University of Florence, available at the following link www.dsa.unifi.it. This website section is managed by CESPD (Centre for Research on Disability Issues) and was developed during the project "Students with LD at the University. Implementation of a methodological protocol for teaching usability and self-study" co-financed by Fondazione Cassa di Risparmio di Firenze and developed by the Centre for Research on Disability Issues, by the Department of Industrial Engineering and the Department of Education and Psychology. From the homepage, it is possible to access the following sections:

- LD Description;

- Facilities;

- Instruments;

- Activities for LD students:

- Contact information.

The "Facilities" section includes a part dedicated to "Teachers Facilities", divided in the following sections:

- Guidelines;

- Handbook;

- Instruments;

- Training.

Guidelines: they are in digital format and can be downloaded from the website. Teachers, students and technical and administrative staff can find a dedicated specific section, containing best practices and operational guidance.

A concise handbook with basic instructions for the management of students with LD. The teachers Handbook can be downloaded from the website as well.

An E-Learning course on Moodle platform dedicated to LD aimed to inform and sensitize the different actors involved in the management of students with LD within the University of Florence. Among its various materials, the course also contains a video-tutorial on the Guidelines and their use.

\section{Concluding remarks}

In conclusion, the Guidelines for the didactic and inclusion of LD students of the University of Florence are articulated around a global and systemic plan of action, meant to accompany and protect the students during their study career, even before enrolling at the University, with four different declination: 
1. The information and logistics level, useful for the student to access easily all the information necessary to find the resources and supports provided by the University;

2. The level of the relational aspects, related to the support given from LD teachers delegates and educational process tutors;

3. The level relating to aspects connected with teaching fruition (use of technological instruments, adoption of methodologies, preparation of appropriate teaching materials, examination performing methods);

4. The level of support to individual study, provided by the teacher or tutor (for example the construction of customized materials, or the retrieval of study methods, etc.).

It is therefore a systemic intervention plan for LD students, which roused and keeps rousing the interest of all the university system, with a radical consideration on academic teaching, since while we try to provide the best LDfriendly didactic in compliance with the rules, no one can be exempted from a wider consideration on the nature and the quality of university teaching offered to all students.

\section{References}

aDShe - Association of Dyslexia Specialist in Higher Education (2011), "Guidance for Good Practice: Reasonable Adjustments", http://tinyurl.com/psv2x8r.

Aiutodislessia (2013), "Linee Guida per la leggibilità", http://tinyurl.com/q7hhrvs.

British Dyslexia Association (2015), "Dyslexia Style Guide", http://tinyurl.com/nbg4yye.

British Dyslexia Association (2015), "Typefaces for dyslexia", C B.D.A. New Technologies Committee, http://tinyurl.com/nur8ubo.

CAST (2011), "Universal design for learning guidelines version 2.0", Wakefield, MA: (C) 2011 by CAST, http://tinyurl.com/yeskk49.

CNUDD, Conferenza Nazionale Universitaria Delegati per la Disabilità, Linee guida, 10 luglio 2014, http://tinyurl.com/nvew2yk.

Dehaene S. (2009), "I neuroni della lettura", Milano, Raffaello Cortina.

Disabled Students' Program (2011), Berkeley, University of California, "Teaching Students with Disabilities", UC Berkeley: Copyright (C) 2011-14 UC Regents, http://tinyurl.com/nb3n5h7.

DO-IT (2012), “Academic Accommodations for Students with Learning Disabilities: Achieving equal access through accommodations and universal design", (C) 2012, 2009, 2007, 2004, University of Washington, http://inyurl.com/o3bdodh.

Genovese E., Ghidoni E., Guaraldi G. (2011), "Dislessia nei giovani adulti”, Trento, Erickson.

Gérard C.-L. (2011), “Cliniques des troubles des apprentissages. De l'évaluation neuropsychologique à la programmation éducative", Bruxelles, De Boeck. 
Hatcher J. et al. (2002), "Cognitive assessment of dyslexic students in higher education", British Journal of educational Psychology, 72, 119-133.

Lami L. et al. (2008), "Evoluzione del profilo di lettura della dislessia. Studio longitudinale su un gruppo di dislessici divenuti giovani adulti”, Dislessia, 1, 7-17.

Legge 8 ottobre 2010, n. 170 "Nuove norme in materia di disturbi specifici di apprendimento in ambito scolastico", Gazzetta Ufficiale N. 244, 18 Ottobre 2010.

Meyer A., Rose D.H., \& Gordon D. (2014), "Universal design for learning: Theory and practice", Wakefield MA:CAST, http://tinyurl.com/o4gvk3n.

CNUDD - Conferenza Nazionale Universitaria Delegati per la Disabilità (2014), "Linee guida", 10 luglio 2014, http://tinyurl.com/nvcw2yk.

Ministero dell'Istruzione, dell'Università e della Ricerca Dipartimento per l'Istruzione Direzione Generale per lo Studente, l'Integrazione, la Partecipazione e la Comunicazione, "Linee guida per il diritto allo studio degli alunni e degli studenti con disturbi specifici di apprendimento", allegate al decreto ministeriale 12 luglio 2011.

Olofsson A. et al. (2012), "Learning and study strategies in university students with dyslexia: implications for teaching", Procedia Social and behavorial Sciences, 47, 1184-1193.

Reid A.A. et al. (2006), "Cognitive Profiles of adult developmental Dyslexics:Theoretical implications", Wiley InterScience, 13, 1-24.

Reid G., Strnadová I., and Cumming T. (2013), "Expanding horizons for students with dyslexia in the 21 st century: universal design and mobile technology", Journal of Research in Special Educational Needs, Vol. 13, N. 3, pp. 175-181, doi: 10.1111/1471-3802.12013.

Rello L. and Baeza-Yates R. (2012), “Optimal colors to improve readability for people with dyslexia" in Text customatization for readability on line Symposium, 19 november 2012, : http://tinyurl.com/om6y84o.

Rello L. and Baeza-Yates R. (2015), "Good Fonts for Dyslexia" in ASSETS '13 Proceedings of the 15th International ACM SIGACCESS Conference on Computers and Accessibility, Article No. 14 / ACM New York, NY, USA C2013 ISBN: 978-14503-2405-2 /doi >10.1145/2513383.2513447.

The Ohio State University Partnership Grant (2015), "Improving the Quality of Education for Students with Disabilities", Fast fact for faculty. Universal design for learning: Elements of good teaching, http://tinyurl.com/qz5d3m2.

Trisciuzzi L. and Zappaterra T. (2012; 2014), "La dislessia. Una didattica speciale per le difficoltà di lettura e scrittura", Milano, Guerini e Associati.

University of Pennsylvania, Weingarten Learning Resources Center (2002), "Teaching Students Who Have a Learning Disability: Strategies for Faculty, Tutors, and Learning Instructors", http://tinyurl.com/oxrurve.

Zappaterra T. (2012), "La lettura non è un ostacolo. Scuola e DSA”, Pisa, ETS.

Zecchi-Orlandini S., Zappaterra T., Campatelli G., Ariani L., Meneghin A., Rossi C., Zudetich C. (2015), "Students with Learning Disabilities at University. Design of a Protocol for Usability of Teaching and Individual Study", Italian Journal of Special Education for Inclusion, 1, 2015, 125-136. 